\title{
Transition metal on topological chiral semimetal PdGa with tailored hydrogen adsorption and reduction
}

\author{
Qun Yang ${ }^{1}$, Guowei Li $\mathbb{D}^{1,2,3 凶}$, Yudi Zhang ${ }^{2,3}$, Jian Liu $^{2,3}$, Jiancun Rao $\mathbb{D}^{4}$, Thomas Heine $\mathbb{D}^{5}$, Claudia Felser ${ }^{1}$ and Yan Sun $\mathbb{D}^{1 凶}$
}

The difficulties in designing high-performance hydrogen evolution reaction (HER) catalysts lie in the manipulation of adsorption behaviors of transition metals (TMs). Topological chiral semimetals with super-long Fermi arc surface states provide an ideal platform for engineering the catalytic performance of TMs through the metal-support interaction. We found the adsorption trends of TMs can be modified significantly when deposited at the surface of the PdGa chiral crystal. The electron transfer from the TMs to the surface states of the PdGa reshapes the $d$ band structure of TMs and weakens the hydrogen intermediate bonding. Especially, W/PdGa is expected to be a good HER catalyst with close to zero Gibbs free energy. Experimentally, we found a Pt-like exchange current density and turnover frequency when depositing $W$ atoms at the PdGa nanostructures surface. The findings provide a way to develop high-efficient electrocatalysts by the interplay between topological surface states and metal-support interaction.

npj Computational Materials (2021)7:207; https://doi.org/10.1038/s41524-021-00684-5

\section{INTRODUCTION}

Topological materials have attracted extensive attention in the fields of chemistry and materials sciences owing to their robust topological surface states (TSSs) and high-mobility electrons. Benefited from the bulk symmetry-protected band structure and surface properties, topological materials are considered as a class of promising candidates for catalytic reactions such as hydrogen evolution, $\mathrm{CO}$ oxidation, and $\mathrm{CO}_{2}$ electroreduction. ${ }^{1-7}$ Among them, topological chiral semimetal sharing the noncentrosymmetric cubic B20 structure is more attractive because of the experimentally confirmed super-long Fermi arcs that cross the entire Brillouin zone. ${ }^{6,8-10}$ As HER catalysts, their topological surface states interact directly with hydrogen intermediates, exhibiting impressive high activities. This class of topological chiral semimetals has large non-trivial energy windows up to $1 \mathrm{eV}$, and more importantly, $80 \%$ of the surface states are localized at the first atomic layer. Thus, they constitute a family of highperformance catalysts and also provide an ideal platform for studying the synergistic effects of substrates.

Supported metal catalysts are commonly used for many industrial chemical reactions as they can significantly boost the activities of supported metals. ${ }^{11-16}$ On one hand, the support is used to stabilize the dispersed metal to achieve a long catalyst life and low cost. On the other hand, the support can induce substantial electronic perturbations to the anchored metal catalysts by modifying their morphology, electronic structure, and catalytic performance. ${ }^{17-19}$ This sets off a boom-time for the effective design of single or binary-atom catalysts on various supports such as graphene, graphite, and metal oxides under the principle of metal-support interaction (MSI). ${ }^{20}$ Recently, topological materials were proposed as a new family of supports for catalysts, which could tune the adsorption and catalysis behaviors because of the electron transfer from the robust surface states. ${ }^{2,4-7,21}$ However, traditional topological insulators, like $\mathrm{Bi}_{2}(\mathrm{Se} / \mathrm{Te})_{3}$, either have a narrow nontrivial energy window or are chemically unstable merging in the solutions over a long time. ${ }^{22,23}$ Such disadvantages block their further experimental analysis and applications as catalysts in HER. Therefore, topological materials with both robust TSSs and chemical stability in the HER process are desirable. Transition metal-based topological chiral semimetals fulfill such requirements.

By combining the first-principles calculations and experimental measurements, we find that topological chiral semimetals, such as PdGa, could be a catalog of support that can greatly modify the adsorption behaviors of the supported metal catalysts of Mo, W, $\mathrm{Ru}, \mathrm{Rh}, \mathrm{Pd}, \mathrm{Pt}, \mathrm{Ni}, \mathrm{Co}$, and Fe via MSI. In comparison with these pure metal catalysts that located on the left of the HER volcano plot due to strong hydrogen intermediate binding strength, the adsorption energies of hydrogen intermediate on these metal catalysts that dispersed on PdGa support can be weakened significantly, which is attributed to the interaction between the TSSs and deposited metals. Thus, TM/PdGa catalysts are all located on the right of the volcano plot. In particular, we find that W/PdGa is a promising HER catalyst with Pt-like activity. Different from the pristine PdGa, which is inert for hydrogen evolution due to the low density of states around the Fermi level. The MSI between PdGa Fermi arcs and the supported $\mathrm{W}$ catalyst leads to a high intensity atomically narrow $\mathrm{W}-5 d$ electron locating at the Fermi level. These narrow $\mathrm{W}-5 d$ states can intrinsically increase the $\mathrm{H}$ intermediate binding, thereby exhibiting high intrinsic activity. The finding in this work reveals a valuable method to develop an efficient HER electrocatalyst by manipulating the MSI based on topological materials.

\section{RESULTS AND DISCUSSION}

Fermi arc surface states in topological chiral crystal

PdGa is a structurally chiral topological semimetal (see Fig. 1a), which belongs to the non-symmorphic chiral space group $\mathrm{P} 2{ }_{1} 3$ (number 198), with Pd and $\mathrm{Ga}$ atoms forming right- and

\footnotetext{
${ }^{1}$ Max Planck Institute for Chemical Physics of Solids, 01187 Dresden, Germany. ${ }^{2}$ CAS Key Laboratory of Magnetic Materials and Devices, and Zhejiang Province Key Laboratory of Magnetic Materials and Application Technology, Ningbo Institute of Materials Technology and Engineering, Chinese Academy of Sciences, Ningbo 315201 , China. ${ }^{3}$ University of Chinese Academy of Sciences, 19 A Yuquan Rd, Shijingshan District, Beijing 100049, China. ${ }^{4}$ AIM Lab, Maryland NanoCenter, University of Maryland, College Park, MD 20742, USA.

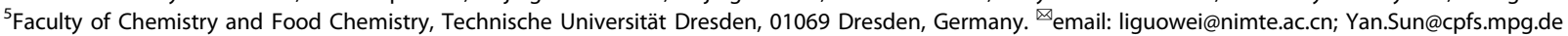




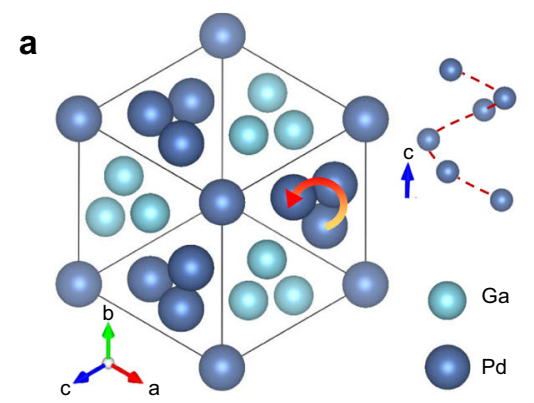

d

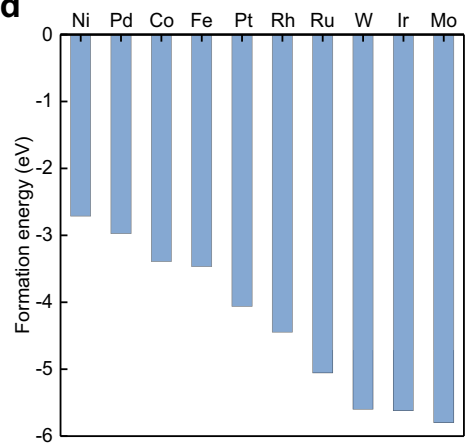

b

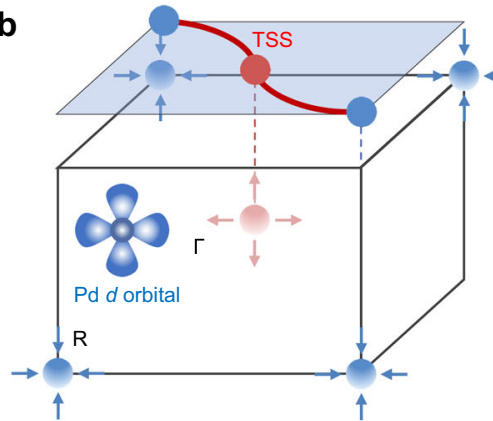

e

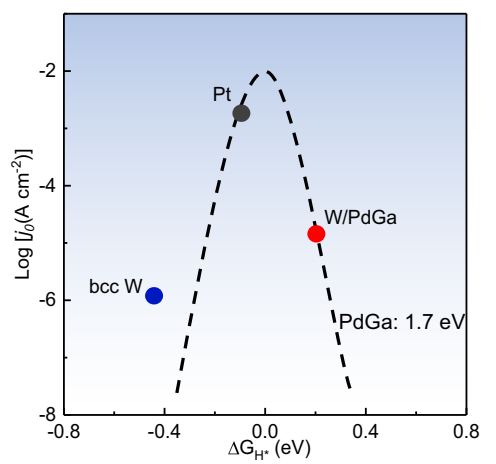

C

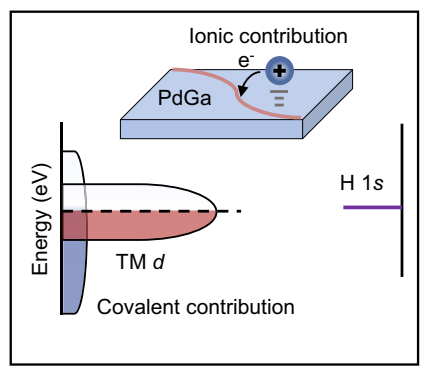

f

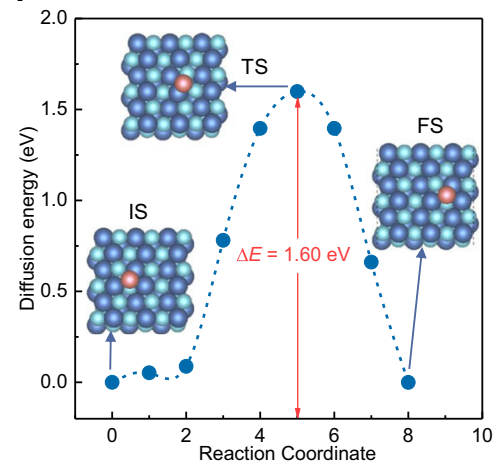

Fig. 1 Geometric, electronic, and catalytic properties of topological chiral crystal PdGa. a Crystal structures of PdGa in the view of [111] direction. The right-helices formed by Pd are shown in the right-upper inset. b Bulk BZ and surface (001) BZ with Pd-d-orbital-derived Fermi arcs connecting the projections of $\mathrm{R}$ and $\Gamma$ points. $\mathrm{c}$ Metal-support interaction modification of the electronic structure of supported metal catalyst for HER. d Formation energies of single TM atom anchored on the PdGa (001) surface. e Gibbs free energy $\left(\Delta G_{H^{*}}\right)$ of pristine PdGa and W/PdGa catalysts for hydrogen evolution. The $\Delta G_{\mathrm{H}^{*}}$ for pure metal catalysts of Pt and W are also included for comparison. ${ }^{25,32} \mathbf{f}$ Energy barrier for the W ion diffusion on the PdGa (001) surface. The insets are initial state, transition state, and final state, respectively.

left-handed helices, respectively. Protected by the crystal symmetry and $\mathrm{Pd}-d$ orbitals around Fermi level, two multifold chiral fermions with a large topological charge of Chern number 4 and -4 locate at the high symmetry momenta of $\Gamma$ and $R$, respectively, see Fig. $1 \mathrm{~b}$. It, therefore, renders Fermi arcs to span over the entire surface BZ and topological bands to straddle giant nontrivial energy windows of $1.869 \mathrm{eV}$, which can present as an effective electron donor or acceptor, as illustrated in Fig. 1c. ${ }^{6,8,24}$ Besides, such large partial-filled TSSs could possibly maximize the topological effect on the supported metal catalyst. Most importantly, PdGa is known to be chemically inert during electrochemical HER, which is extremely important for clarifying the contributions from MSI. ${ }^{6}$

\section{Stabilities and catalytic activities}

With this motivation, we investigated the single atoms of typical transition metals (TMs) ${ }^{25-28}$ (including $\mathrm{Mo}, \mathrm{W}, \mathrm{Ru}, \mathrm{Rh}, \mathrm{Pd}, \mathrm{Pt}, \mathrm{Ni}, \mathrm{Co}$, and Fe) supported on the PdGa. First, we checked the stability of the TM/PdGa by considering all the possible high symmetry sites of PdGa for anchoring the isolated TM atom. The favorable sites for TM atoms immobilization can be classified into two categories. As shown in Supplementary Fig. 1, the isolated Mo and W atoms are preferred to stay on the bridge site of the $\mathrm{Pd}-\mathrm{Ga}$ bond, while the energetically most favorable binding site for $\mathrm{Ru}, \mathrm{Rh}, \mathrm{Pd}, \mathrm{Pt}, \mathrm{Ni}$, $\mathrm{Co}$, and Fe is near the top $\mathrm{Ga}$ atom. As presented in Fig. 1d, the large negative formation energies, (especially for $\mathrm{Mo}, \mathrm{W}, \mathrm{Ru}$, and Ir with $E_{\text {formation }}<5 \mathrm{eV}$ ) suggest that these TMs could bond stably with the PdGa support. Furthermore, to investigate whether the surface HER conditions can influence the stability of isolated TM on PdGa, the stability of W/PdGa was further explored with the inclusion of the solvation effect in the calculation. By using the implicit solvent model implemented in VASPsol with a dielectric constant of $80^{29}$, the formation energy of W/PdGa was calculated to be $-6.21 \mathrm{eV}$, indicating that W/PdGa is still stable under HER conditions.

With this starting point, the catalytic activities of all the PdGa supported metal catalysts were evaluated by the Gibbs free energies of the $\mathrm{H}$ intermediate $\left(\left|\Delta G_{\mathrm{H}^{*}}\right|\right) \cdot{ }^{25,30,31}$ As shown in Fig. 1e and Supplementary Fig. 3, a large positive $\Delta G_{\mathrm{H}^{*}}$ of $1.703 \mathrm{eV}$ for its most stable configuration on pristine $\mathrm{PdGa}$ suggests the chemically inert nature towards $\mathrm{H}$ intermediate. The presence of MSI greatly modifies the catalytic performance of supported metal catalysts. Unlike most of the pure metal catalysts with negative $\Delta G_{H^{*}}$, all the supported metal catalysts on PdGa have a positive value for $\Delta G_{\mathrm{H}^{*}}$. Especially for W/PdGa, it shows a close to zero $\Delta G_{\mathrm{H}^{*}}$ of $0.2 \mathrm{eV}$, comparable to that in the ideal Pt (111) catalyst $\left(\left|\Delta G_{\mathrm{H}^{*}}\right|\right.$ $=0.09 \mathrm{eV}) .{ }^{32,33}$ Therefore, a superior HER activity of W/PdGa is expected from the thermodynamics point of view, which should be better than that of $\mathrm{Pt} / \mathrm{PdG}$ catalyst with a larger $\Delta \mathrm{G}_{\mathrm{H}^{*}}$ value of $0.741 \mathrm{eV}$. In this work, W/PdGa and Pt/PdGa catalysts will be synthesized and analyzed to prove our hypothesis.

During the deposition of TM atoms on the substrate, the actual behavior of the system will depend on not only the energetics of the configurations but also the potential barriers, temperature, and deposition rate. By using the climb-image nudged elastic band method, the minimum-energy pathway for the $\mathrm{W}$ atom migration between two adjacent energetic most favorable sites was calculated. As displayed in Fig. 1f, the transition barrier for the $\mathrm{W}$ migration is $1.6 \mathrm{eV}$, which is large enough to prevent aggregation of the isolated $W$ atoms, suggesting that the supported W atom can hardly diffuse to form W clusters on PdGa.

\section{Catalytic activity origin study}

To confirm the crucial role of PdGa support for the excellent catalytic performance of W/PdGa catalyst and to understand the interaction mechanism, we firstly investigated the surface 


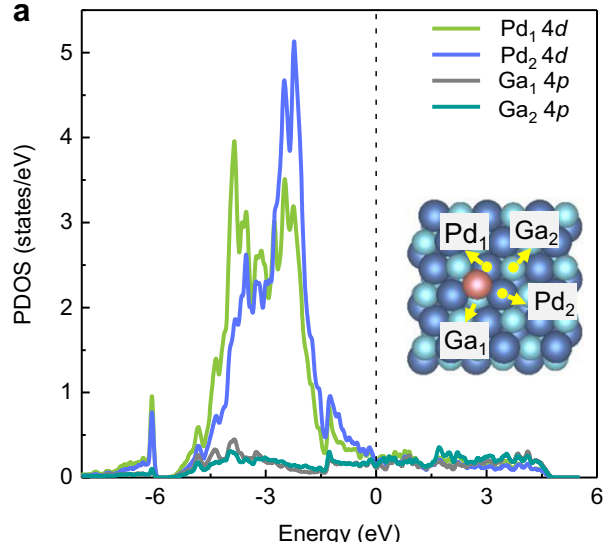

C

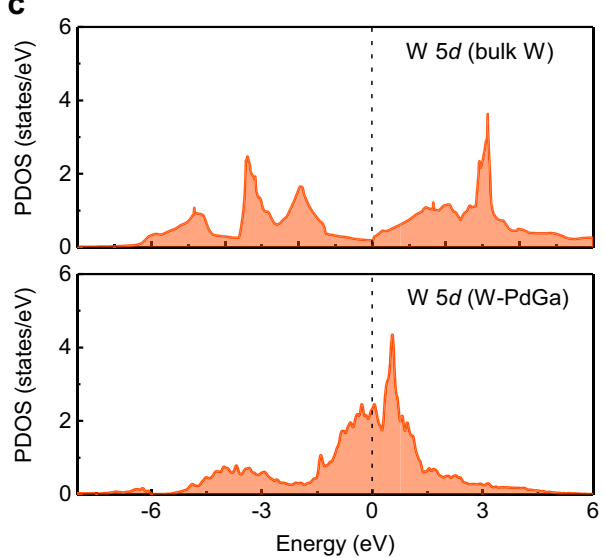

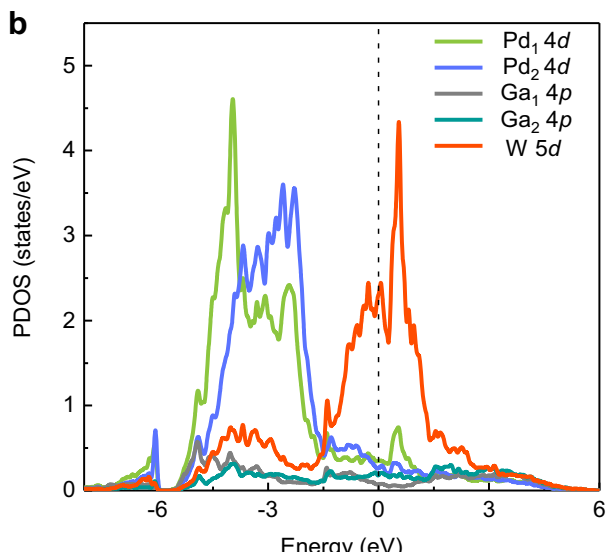

d

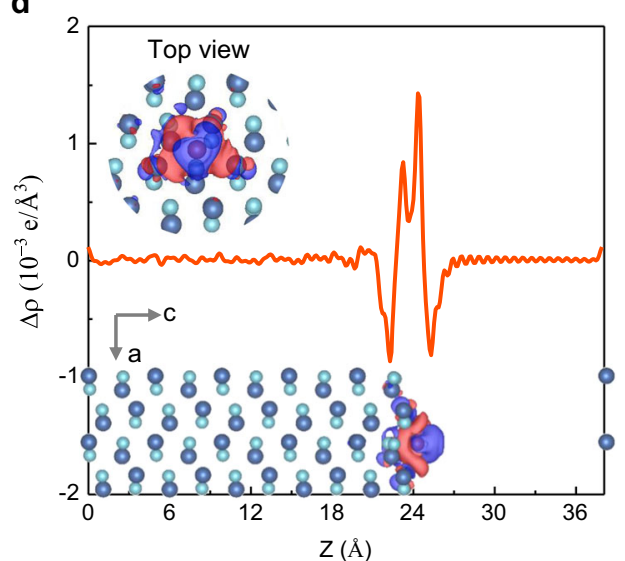

Fig. 2 Surface electronic structure of W/PdGa. Projected DOS of $4 d, 4 d, 4 p, 4 p$ orbitals of surface $\mathrm{Pd}_{1}, \mathrm{Pd}_{2}, \mathrm{Ga}_{1}$, and $\mathrm{Ga}_{2}$ atoms (as indicated in the inset of Fig. 3a) a before and (b), after interaction with W. c Calculated W $5 d$ DOS in bulk bcc W and W/PdGa catalyst, respectively. d The plane averaged charge density difference (CDD) for W/PdGa catalyst. The positive and negative $\Delta \rho$ mean the electron accumulation and depletion, respectively. The top and side view of visualized CDD with an isosurface value of $0.002 \mathrm{e} / \AA^{3}$ are also shown in the inset. The red and blue areas represent the electron accumulation and depletion, respectively.

properties of the W/PdGa by projected density of states. As indicated in Fig. 2a-c, the pristine PdGa is a semimetal with a low density of states around the Fermi level, corresponding to the unfavorable adsorption behavior of $\mathrm{H}$. The electronic structure changes dramatically after $\mathrm{W}$ anchoring on PdGa. It displays a greatly improved $d$-electron domination around the Fermi level. Compared with bulk BCC-W, the W-5d states in W/PdGa catalyst are atomically narrow. Most importantly, they are localized around the Fermi level, which makes bonding with adsorbate energetically more preferred. From the hybridization of DOS in Fig. 2b, this localized W $5 d$ state near the Fermi level comes from its weak interaction with the electronic states of the PdGa support. Such atomically narrow $d$ states arising from the weak interaction between substrate and its supported metal catalyst were also recently confirmed in some other studies. ${ }^{28,34,35}$ Subsequently, the surface states of pristine PdGa and W/PdGa were investigated to understand the effect of TSSs on electronic states engineering of the deposited W. In this case, a $1 \times 1$ supercell of PdGa (001) slab model with ten unit cells thickness was constructed as the support for study, which can capture the main physical feature with different W-coverage. As shown in Supplementary Fig. $4 a$, the surface states of pristine PdGa are mostly dominated by the Fermi arc surface states across the whole surface Brillouin zone. Moreover, these Fermi arc surface states are partially occupied and straddle a large energy window around the Fermi level (Supplementary Fig. 4b), which are active and can act as the electron donor or acceptor for the deposited W. After the W deposition, the shape of Fermi arcs surface states change dramatically (Supplementary Fig. 4c). They shift down by accepting the electrons provided by the isolated $\mathrm{W}$ atom (Supplementary Fig. 4d).

It is well known that ionic and covalent bonding dominate the binding trends between the substrate and adsorbate. Based on the Bader charge analysis, ${ }^{36}$ we found that the $\mathrm{W}$ atom loses $0.352 \mathrm{e}^{-}$ electrons to Fermi arc surface states of PdGa support. It, therefore, causes an evident charge redistribution with $\mathrm{W}$ becoming the charge depletion center (Fig. 2d). Consequently, the supported W catalyst is positively charged with cation-like states, which influences its ionic bonding characteristic with an adsorbate. Apart from that, the presence of the narrow $\mathrm{W} 5 d$ states reflects the possible strong interaction with the hydrogen $1 \mathrm{~s}$ orbital, which makes the bonding between the $\mathrm{W}$ and hydrogen exhibit more covalent bonding characteristics. Therefore, the PdGa supportbased heterogeneous catalyst inherits the exceptional selectively virtue of homogeneous catalysts.

The narrow $5 d$ states of the supported cation-like high-valence W catalyst are chemically active and can interact strongly with the $\mathrm{H}$ intermediate. After $\mathrm{H}$ absorption, the $\mathrm{W}-5 d$ states change a lot. The DOS from W-5d orbitals at the Fermi level decreases, and the occupied $\mathrm{H}-1 \mathrm{~s}$ orbitals present in the energy region from $-4 \mathrm{eV}$ to $-2 \mathrm{eV}$. Moreover, the evident orbital hybridization occurs between the $\mathrm{H}-1 \mathrm{~s}$ and $\mathrm{W}-5 d$ orbitals in this energy region, as shown in Fig. 3a. Besides, the orbital projected DOS of W before and after interaction with $\mathrm{H}$ demonstrates that the $\mathrm{W}-5 d_{\mathrm{z}^{2}}$ and $\mathrm{W}-5 d_{\mathrm{xz}}$ related states dominate the reaction with $\mathrm{H}-1 \mathrm{~s}$ orbital, owing to their suitable orbital orientation (Supplementary Fig. 5 and Fig. 3b). Furthermore, charge density differences in Fig. $3 \mathrm{C}$ and Bader charge analysis show that the adsorbed $\mathrm{H}$ 

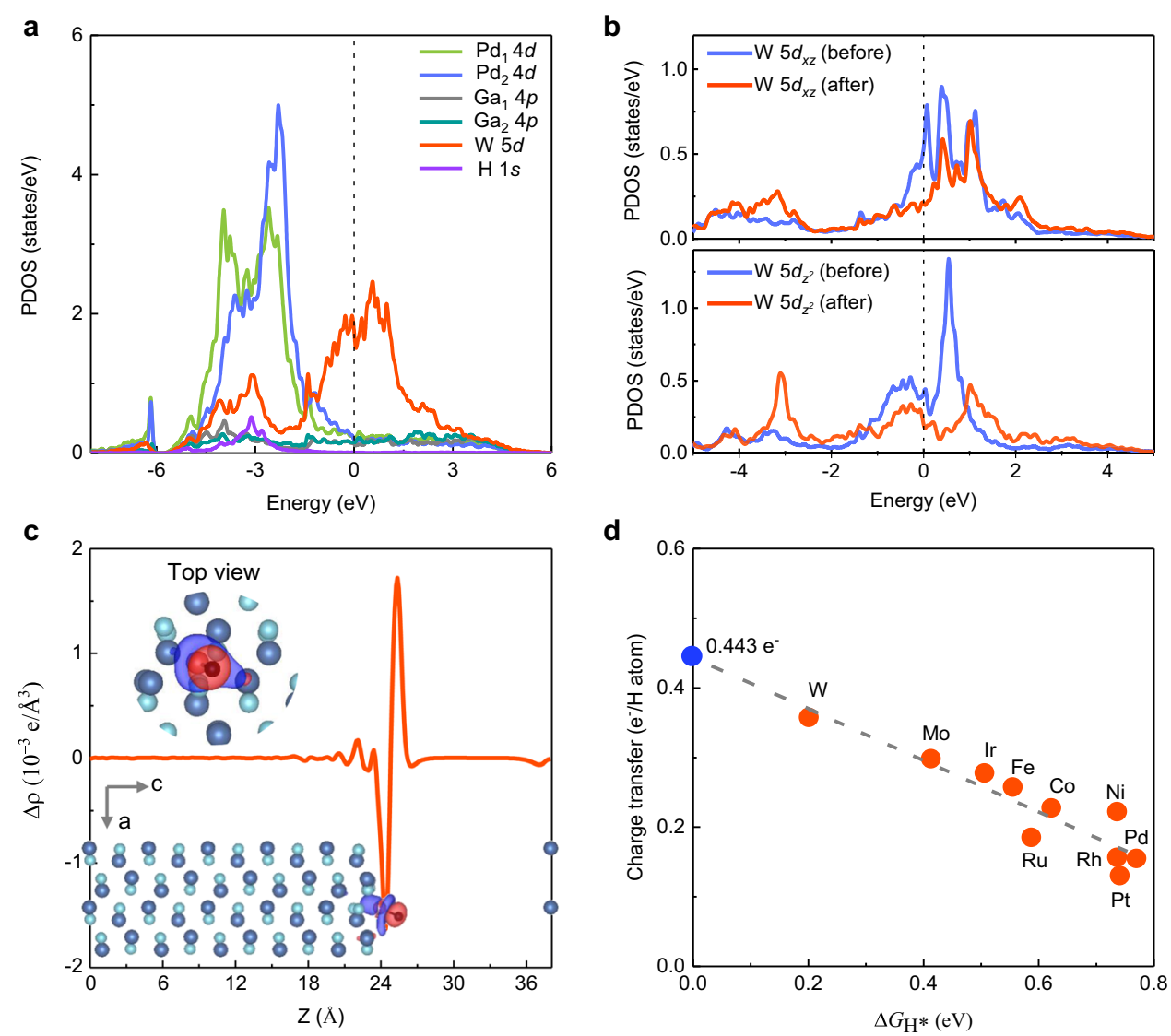

Fig. 3 Surface electronic structure of W/PdGa after $\mathbf{H}$ adsorption. a Projected DOS of W/PdGa catalyst after $\mathbf{H}$ adsorption. $\mathbf{b}$ Projected DOS of the active $\mathrm{W}-5 d_{\mathrm{xz}}$ and $\mathrm{W}-5 d_{\mathrm{z}^{2}}$ orbitals of W/PdGa catalyst before and after interacting with the $\mathrm{H}$ intermediate. $\mathrm{c}$ The plane averaged charge density difference (CDD) for $\mathrm{H}$ adsorbed W/PdGa system. The positive and negative $\Delta \rho$ mean the electron accumulation and depletion, respectively. The top and side views of visualized CDD with an isosurface value of $0.002 \mathrm{e} / \AA^{3}$ are also shown in the inset. d Relationship between Bader charge transfer and HER activity $\left(\Delta G_{\mathrm{H}^{*}}\right)$ of all the studied TM/PdGa catalysts. The ideal charge transfer with $\Delta G_{\mathrm{H}^{*}}=0 \mathrm{eV}$ is predicted to be $0.443 \mathrm{e}^{-}$as labeled by the blue circle in the figure.

obtains charges from the supported W catalyst. Therefore, charge accumulation happens around the adsorbed $\mathrm{H}$ with $\mathrm{S}$ orbital shape, while the charge depletion occurs on the supported W catalyst. In total, a large number of charges up to $0.358 \mathrm{e}^{-}$is transferred from the $\mathrm{W}$ to adsorbed $\mathrm{H}$ intermediate.

The charge transfer could behave as the possible descriptor to quantify the bonding strength between adsorbate and the supported metal catalyst. To explore the activity relation, the Bader charge transfer was calculated and plotted as the function of the $\Delta G_{H^{*}}$ for all supported metal catalysts, as shown in Fig. 3d. A maximum charge transfer to adsorbed $\mathrm{H}\left(0.358 \mathrm{e}^{-} / \mathrm{H}\right.$ atom) was found for W/PdGa system, corresponding to the strongest binding energy and best HER activity $\left(\Delta G_{H^{*}}=0.20 \mathrm{eV}\right)$. The number of charge transfer displays an almost linear correlation with the $\Delta G_{H^{*}}$, with a few of them ( $\mathrm{Ru}, \mathrm{Ni}, \mathrm{Pt})$ slightly deviated. The obtained linear relationship suggests an ideal charge transfer $\left(0.443 \mathrm{e}^{-} / \mathrm{H}\right.$ atoms approximately) to design a highly active TM/PdGa catalyst for HER.

\section{Electrochemical behaviors for hydrogen evolution}

To verify our prediction, we synthesized PdGa nanostructures by a solid states reaction procedure. Thereafter, $\mathrm{W}$ and $\mathrm{Pt}$ single atoms with a very low concentration $(\sim 1.1 \mathrm{wt} \%$ and $1.5 \mathrm{wt} \%$, respectively.) were loaded according to previous reports (see details in experimental section) to avoid agglomeration. ${ }^{37-39}$ The phase and crystallinity of the prepared PdGa carriers were verified by powder X-ray diffraction (XRD) and scanning electron microscopy (SEM) (Supplementary Figs. 6 and 7). Subsequently, the prepared PdGa carriers were mixed with $\mathrm{Na}_{2} \mathrm{WO} \cdot 2 \mathrm{H}_{2} \mathrm{O}$ and $\mathrm{H}_{2} \mathrm{PtCl}_{6} \cdot 6 \mathrm{H}_{2} \mathrm{O}$ solutions, to produce the supported W (W/PdGa) and $\mathrm{Pt}(\mathrm{Pt} / \mathrm{PdGa})$ catalysts. The successful anchoring of $\mathrm{W}$ atoms is revealed by the corresponding SEM (Supplementary Fig. 8) and EDS elemental mapping (Supplementary Fig. 9). The ultra-small size of $\mathrm{W}$ species is further confirmed by the high-angle annular darkfield scanning transmission electron microscopy coupled with elemental mapping (Supplementary Fig. 10), although it is still difficult to see the single $\mathrm{W}$ atoms directly due to the large size of PdGa support. The HER catalytic activity of the investigated catalysts was evaluated using a standard three-electrode electrochemical cell in $0.5 \mathrm{M} \mathrm{H}_{2} \mathrm{SO}_{4}$. The catalyst mass loading on a glassy carbon electrode was approximately $0.5 \mathrm{mg} \mathrm{cm}^{-2}$. The linearsweep voltammograms (LSVs) after iR-compensation for the $20 \%$ $\mathrm{Pt} / \mathrm{C}, \mathrm{PdGa}$, and W/PdGa catalysts are displayed in Fig. $4 \mathrm{a}$. Pt/C catalyst exhibits superior HER catalytic activity with a near-zero onset potential, as well as a low overpotential of $23 \mathrm{mV}$ to reach a current density of $10 \mathrm{~mA} \mathrm{~cm} \mathrm{~cm}^{-2}$. Expectedly, PdGa nanostructured catalysts gave poor HER performance with a large overpotential of more than $290 \mathrm{mV}$ at $10 \mathrm{~mA} \mathrm{~cm}{ }^{-2}$ owing to its unfavorable $\mathrm{H}$ intermediate bonding strength. In contrast, the W/PdGa catalyst exhibits a greatly optimized catalytic activity, giving a low overpotential of $97 \mathrm{mV}$ to produce the same cathodic geometric current density. Moreover, the W/PdGa catalyst achieved a Tafel slope of $78 \mathrm{mV} / \mathrm{dec}$, much smaller than that of PdGa $(111 \mathrm{mV} / \mathrm{dec})$ (Fig. 4b), indicating the improved reaction kinetics and better HER activity in the presence of PdGa substrate. ${ }^{40}$ The above merits of 

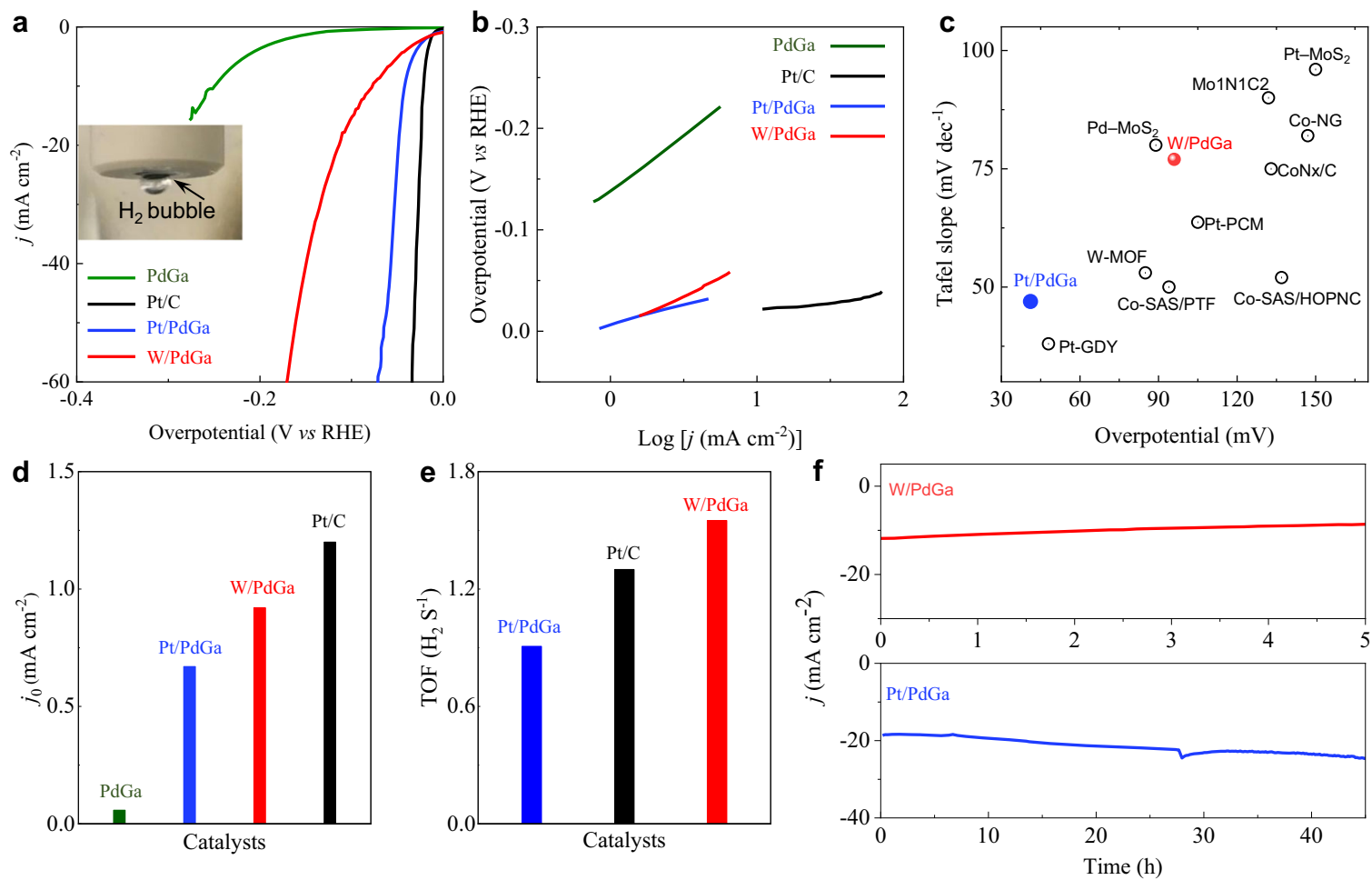

Fig. 4 Catalytic behaviors of W/PdGa and Pt/PdGa HER catalysts. a LSV polarization curves of PdGa, 20\% Pt/C, Pt/PdGa, and W/PdGa catalysts (with iR correction). b Tafel plots derived from the results given in Fig. 2a. c Overpotential and Tafel slopes of W/PdGa and Pt/PdGa catalysts in comparison with other representative catalysts. $d$ Exchange current density for the PdGa, 20\% Pt/C, Pt/PdGa, and W/PdGa samples derived from the Tafel plots. e Comparison of TOF values of $20 \% \mathrm{Pt} / \mathrm{C}, \mathrm{Pt} / \mathrm{PdGa}$, and W/PdGa catalysts. f Stability assessment of Pt/PdGa and W/PdGa catalysts.

the W/PdGa, including low overpotential and Tafel slope, is superior to most previously reported earth-abundant single-atom catalyst (SAC) and even comparable with some Pt-based catalysts (Fig. 4c). ${ }^{41-43}$ This demonstrates the vital role of PdGa carrier to the HER activity of W sites.

For a fair comparison of the HER catalytic activity, the polarization curves were normalized by the electrochemical surface areas (ECSA). The ECSAs were obtained by testing the double-layer capacitance $\left(C_{\mathrm{dll}}\right)$ of the corresponding catalysts. The value for $\mathrm{Pt} / \mathrm{C}, \mathrm{Pt} / \mathrm{PdGa}$, and $\mathrm{W} / \mathrm{PdGa}$ is $0.4,2.5$, and $3.55 \mathrm{mF} \mathrm{cm}^{-2}$, respectively (Supplementary Figs. 11-13). This indicates a much smaller electrochemically active surface area for the catalysts with PdGa carrier. The results are not surprising because of the low loading of $W$ elements on PdGa carriers. As shown in Supplementary Fig. 14, the specific activity of W/PdGa is comparable with the state-of-the-art Pt/C catalysts when scaling the current with ECSAs. The exchange current densities (jo) were calculated to demonstrate the high inherent HER activity (Fig. 4d). This value for W/PdGa was determined to be $0.92 \mathrm{~mA} \mathrm{~cm}{ }^{-2}$, which was close to that of $\mathrm{Pt} / \mathrm{C}\left(1.2 \mathrm{~mA} \mathrm{~cm}{ }^{-2}\right)$ and significantly larger than that of PdGa carrier $\left(0.06 \mathrm{~mA} \mathrm{~cm}^{-2}\right)$ and the other studied precious SACs. ${ }^{42}$ Distinctly, W/PdGa gives a remarkable high turnover frequency (TOFs) of $1.6 \mathrm{~s}^{-1}$, even higher than that of Pt/C $\left(1.3 \mathrm{~s}^{-1}\right)$, representing one of the best solid-state earth-abundant catalysts. $^{44-47}$. Finally, the electrochemical stability of W/PdGa and $\mathrm{Pt} / \mathrm{C}$ catalysts was investigated by the current-time (i-t) test at a constant overpotential (Fig. 4f). W/PdGa has extremely high stability with negligible decay in HER performance in $5 \mathrm{~h}$. LSV curve was recorded after stability, without significant loss of activity in comparison with the fresh sample (Supplementary Fig. 15).

In the next step, we studied the catalytic behavior of Pt SAC with PdGa as the supporting material. It has been generally accepted that Pt single atoms should be promising catalysts in regardless of the used substrates. ${ }^{48}$ However, our theory suggests that the intrinsic catalytic activity of $\mathrm{Pt} / \mathrm{PdGa}$ should be lower than that of W/PdGa catalyst due to the topological electronic structure of $\mathrm{PdGa}$. Thus, $\mathrm{Pt} / \mathrm{PdGa}$ catalyst was designed following previously reported procedure (Supplementary Fig. 16). ${ }^{39}$ The HER performance was assessed with the same method as for the W/PdGa catalyst. Pt/PdGa exhibited better apparent activities than that of W/PdGa, which requires a low overpotential of $42 \mathrm{mV}$ at the current density of $10 \mathrm{~mA}$ $\mathrm{cm}^{-2}$ (Fig. 4a). However, the exchange current density and TOF value were only $0.672 \mathrm{~mA} \mathrm{~cm}{ }^{-2}$ and $0.907 \mathrm{~s}^{-1}$, respectively, which are lower than W/PdGa (Fig. 4d, e). This indicates a higher intrinsic HER activity of W/PdGa, which is consistent with our theory calculation. All the above merits of investigated catalysts indicate that PdGa carriers can boost the catalytic activity of W atoms, highlighting the vital role of PdGa.

In summary, we have demonstrated that the topological chiral semimetal PdGa can significantly modify the HER performance of supported transition metal catalysts via the MSI approach. The prepared W/PdGa exhibits an impressive turnover frequency of $1.6 \mathrm{~s}^{-1}$, superior to that of the commercial $\mathrm{Pt} / \mathrm{C}$ catalyst. The theoretical calculations reveal that the $\mathrm{W}-5 d$ states in W/PdGa are atomically narrow due to a moderate hybridization with PdGa TSSs. Such hybridization results in a localized positive charge on the $\mathrm{W}$ active site. The modified electronic structure of the supported $W$ catalyst gives rise to favorable bonding energy with the hydrogen intermediate and leads to a high HER catalytic efficiency. This work highlights the importance of topological chiral semimetals as the support to control the MSI for boosting the electrochemical reduction reactions. 


\section{METHODS}

\section{Computational methods}

All our calculations were carried out in the framework of DFT as implemented in the Vienna ab initio simulation package (VASP) ${ }^{49,50}$ The projector augmented wave method was applied to describe the ionelectron interaction, and Perdew-Burke-Ernze-rhof generalized gradient approximation was used to deal with the electron exchange-correlation interaction. ${ }^{51,52}$ To describe the long-range van der Waals interactions between the adsorbed $\mathrm{H}$ and the TM/PdGa catalysts, the DFT-D3 extension of Grimme was adopted..$^{53}$ A $3 \times 3 \times 1$ supercell of PdGa (001) slab model with five unit cells thickness was constructed as the support for anchoring the single TM atom, where the bottom three unit cells were immobilized to simulate the bulk. Noted that this thickness we applied is reasonable as demonstrated by our previous surface energy calculation. ${ }^{6}$ Monkhorst-Pack k-point mesh of $6 \times 6 \times 1$ was used for the geometry optimization and electric characteristic calculations, and the kinetic energy cutoff was set to $400 \mathrm{eV}$. To search a minimal energy path for $\mathrm{W}$ ion diffusion, the climb-image nudged elastic band (CINEB) method embedded in VASP was employed. ${ }^{54}$ The convergence criterion of force was also set to be $0.01 \mathrm{eV} / \AA$, and nine images were used for the CINEB calculations, including the initial and final structural configurations. The stability of TMs immobilized on the PdGa support can be evaluated by calculating the formation energy based on the following formual: ${ }^{55,56}$

$E_{\mathrm{f}}=E_{\mathrm{TM}+\mathrm{PdGa}}-E_{\mathrm{PdGa}}-E_{\mathrm{TM}}$

Where $E_{\mathrm{TM}+\mathrm{PdGa}} E_{\mathrm{PdG}}$ and $E_{\mathrm{TM}}$ are the total energies of TM/PdGa system, pristine PdGa, and isolated TM atom, respectively.

To evaluate the HER catalytic activity of the catalyst, the Gibbs free energy of the $\mathrm{H}$ intermediate adsorbed on the catalyst $\left(\Delta G_{H_{*}}\right)$ can be obtained by the following equations: ${ }^{30}$

$\Delta G_{\mathrm{H}^{*}}=\Delta E_{\mathrm{H}}-\Delta E_{\mathrm{ZPE}}+T \Delta S_{\mathrm{H}}$

Where $\Delta E_{\mathrm{H}}$ is the $\mathrm{H}$ adsorption energy and $\Delta E_{\mathrm{ZPE}}$ and $\Delta S_{\mathrm{H}}$ are the changes in zero-point energy and entropy between the absorbed $\mathrm{H}$ and gaseous $\mathrm{H}$, respectively. The $\Delta E_{Z P E}$ is obtained by:

$\Delta E_{\mathrm{ZPE}}=E_{\mathrm{ZPE}}^{\mathrm{nH}^{*}}-E_{\mathrm{ZPE}}^{(\mathrm{n}-1) \mathrm{H}^{*}}-\frac{1}{2} E_{\mathrm{ZPE}}^{\mathrm{H}_{2}}$

Where the $E_{\mathrm{ZPE}}^{\mathrm{n} \mathrm{H}^{*}}$ and $E_{\mathrm{ZPE}}^{(\mathrm{n}-1) \mathrm{H}^{*}}$ denote the $\mathrm{ZPE}$ of $\mathrm{n}$ and $\mathrm{n}-1$ adsorbed atomic hydrogens on the catalyst without the contribution of the catalyst, respectively. The calculated frequency of $H^{*}$ adsorption on TM/PdGa catalyst is $321.30 \mathrm{meV}$. The calculated frequency of $\mathrm{H}_{2}$ gas is $549.38 \mathrm{meV}$. The $\Delta S_{H}$ is obtained by formula:

$\Delta S_{\mathrm{H}} \cong-\frac{1}{2} S_{\mathrm{H}_{2}}^{0}$

Where the $S_{\mathrm{H}_{2}}^{0}$ is the entropy of $\mathrm{H}_{2}$ gas under the standard condition. Therefore, equation (2) can be rewritten as following:

$\Delta G_{\mathrm{H}^{*}}=\Delta E_{\mathrm{H}}+0.23 \mathrm{eV}$

\section{Experimental procedures}

PdGa synthesis. In a typical synthesis, $0.52 \mathrm{~g} \mathrm{Ga}\left(\mathrm{NO}_{3}\right) \cdot \mathrm{xH}_{2} \mathrm{O}$ and $0.212 \mathrm{~g} \mathrm{Pd}$ powder (200 mesh) were mixed in $25 \mathrm{~mL}$ distilled waters. The solution was stirred overnight and then dried at $80^{\circ} \mathrm{C}$. The obtained mixture was placed in a tube furnace and heated at $750^{\circ} \mathrm{C}$ for $2 \mathrm{~h}$ in Ar. The obtained product was washed with water and ethanol several times.

W/PdGa synthesis. One $\mathrm{mg} \mathrm{Na} \mathrm{Na}_{2} \cdot 2 \mathrm{H}_{2} \mathrm{O}$ and $40 \mathrm{mg}$ of PdGa carrier (corresponding to a W loading of $1.1 \mathrm{wt} \%$ ) were dissolved in $15 \mathrm{~mL}$ of water containing $50 \mathrm{mg}$ of melamine. The mixture was transferred to a Teflonlined autoclave and heated at $190^{\circ} \mathrm{C}$ for 12 hours. The as-prepared sample was washed and dried at $80^{\circ} \mathrm{C}$. The obtained powders were placed in a tube furnace and heated at $800^{\circ} \mathrm{C}$ for $2 \mathrm{~h}$ in $40 \% \mathrm{H}_{2}$ and $60 \% \mathrm{Ar}$.

Pt/PdGa synthesis. $1.59 \mathrm{mg} \mathrm{H}_{2} \mathrm{PtCl}_{6} \cdot 6 \mathrm{H}_{2} \mathrm{O}$ was dissolved in $10 \mathrm{~mL}$ of distilled water and then added dropwise in 15 of distilled water that contains $40 \mathrm{mg}$ of PdGa carrier. The mixture was stirred at $70^{\circ} \mathrm{C}$ overnight and then washed with ethanol repeatedly. Finally, the Pt/PdGa powder was obtained after drying at $60^{\circ} \mathrm{C}$ for $12 \mathrm{~h}$.

Materials characterizations. Powder X-ray diffraction (XRD) data were recorded with a Bruker D8 Advance diffractometer equipped with a $\mathrm{Cu} \mathrm{Ka}$ source $(\lambda=0.15406 \mathrm{~nm})$. The morphologies and structures of the products were characterized with a JEM2100F electron microscope at $200 \mathrm{keV}$. Elemental mapping and analysis were taken on the Bruker EDS with an energy resolution of $126 \mathrm{eV}$.

Electrocatalytic characterization. The electrochemical measurements were performed in a glass three-electrode electrochemical cell on an Autolab PGSTAT302N with an impedance module electrochemistry workstation. A reversible hydrogen electrode $(\mathrm{Ag} / \mathrm{AgCl}(3 \mathrm{M} \mathrm{KCl}))$ and a high surface area graphite rod were used as a reference and counter electrodes, respectively. An RDE with a glassy carbon disk ( $3.0 \mathrm{~mm}$ diameter) served as the working electrodes in evaluating the HER activity. The catalyst ink was prepared by blending $3 \mathrm{mg}$ of each catalyst with $100 \mu \mathrm{l}$ Nafion solution (0.5 wt\%) and $0.4 \mathrm{ml}$ ethanol in an ultrasonic bath. A certain volume of the dispersion was loaded onto a glassy carbon electrode to result in the desirable catalyst loading. The RDE tests were measured in an Ar-saturated $0.5 \mathrm{M} \mathrm{H}_{2} \mathrm{SO}_{4}$ solution at 1600 r.p.m. with a sweep rate of $1 \mathrm{mV} \mathrm{s}^{-1}$. The electrochemical impedance spectroscopy (EIS) measurements were conducted from $100 \mathrm{kHz}$ to $0.1 \mathrm{~Hz}$ with a $5 \mathrm{mV} \mathrm{AC}$ potential. The amplitude of the sinusoidal potential signal was $10 \mathrm{mV}$. The commercial $20 \mathrm{wt} \% \mathrm{Pt} / \mathrm{C}$ catalyst was measured under the same experimental conditions. All potentials were referenced to a reversible hydrogen electrode according to $E($ versus $\mathrm{RHE})=E($ versus $\mathrm{Ag} / \mathrm{AgCl})+(0.210+0.059 \mathrm{pH}) \mathrm{V}$.

Estimation Turnover frequency calculations (TOF). It is suggested that the electrochemical active surface area of the catalyst is linearly proportional to the double-layer capacitance $\left(C_{\mathrm{dl}}\right)$. Thus, we measured the capacitive currents of the $\mathrm{Pt} / \mathrm{C}$ and W/PdGa catalysts in the potential range with no faradic processes happened at various scan rates. The specific capacitance is determined by plotting the capacitive currents as a function of scan rate. The specific capacitance can be converted into an electrochemical active surface area (ECSA) using the specific capacitance value for a flat standard with $1 \mathrm{~cm}^{2}$ of real surface area.

The total number of hydrogen turn-overs was calculated from the current density according to:

$$
\# H_{2}=\left(j \frac{\mathrm{mA}}{\mathrm{cm}^{2}}\right)\left(\frac{1 \mathrm{Cs}^{-1}}{1000 \mathrm{~mA}}\right)\left(\frac{1 \mathrm{~mol} \mathrm{e}^{-}}{96485.3 \mathrm{C}}\right)\left(\frac{1 \mathrm{~mol} \mathrm{H}_{2}}{1 \mathrm{~mole}^{-}}\right)\left(\frac{6.022 \times 10^{23} \mathrm{H}_{2}}{1 \mathrm{~mol}_{2}}\right)=3.12 \times 10^{15} \frac{\mathrm{H} / \mathrm{s}}{\mathrm{cm}^{2}} \mathrm{per} \frac{\mathrm{mA}}{\mathrm{cm}^{2}}
$$

TOF of Pt. We assumed that all the Pt atoms at the surface of the Pt catalysts are active towards HER. Thus the number of active sites per real surface area for Pt can be obtained by:

Pt \# active sites $=\frac{1 \mathrm{~cm}^{-2}}{\left(2.812 \times 10^{-8} \mathrm{~cm}\right) \times\left(2.812 \times 10^{-8} \mathrm{~cm}\right)} \times 2$

Then the HER turnover frequency (TOF) as a function of current density is defined as:

TOF $=\frac{3.12 \times 10^{15} \frac{\mathrm{H}_{2} / \mathrm{s}}{\mathrm{cm}^{2}} \mathrm{per} \frac{\mathrm{mA}}{\mathrm{cm}^{2}} \times|j|}{\# \text { active sites } \times A_{\mathrm{ECSA}}}$

TOF of W/PdGa and Pt/PdGa catalysts. For W/PdGa and Pt/PdGa catalysts, W and Pt atoms are believed as HER active sites according to theoretical calculation results. We assume that all the $\mathrm{W}$ and $\mathrm{Pt}$ atoms are HER active. The number of active sites was calculated from the mass loading on the glassy carbon electrode, the $\mathrm{W}(\mathrm{Pt})$ contents and the $\mathrm{W}(\mathrm{Pt})$ atomic weight. ${ }^{44}$ \#HER active sites:

TOF $=\frac{\text { catalyst loading } \frac{g}{\mathrm{~cm}^{2}} \times \text { catayst mass ratio }(w t \%)}{\text { catalyst molar mass }\left(\frac{\mathrm{g}}{\mathrm{mol}}\right)} \times N_{A}$

Then the HER turnover frequency (TOF) as a function of current density is defined as:

TOF $=\frac{3.12 \times 10^{15} \frac{\mathrm{H}_{2} / \mathrm{s}}{\mathrm{cm}^{2}} \text { per } \frac{\mathrm{mA}}{\mathrm{cm}^{2}} \times|j|}{\# \text { active sites }}$

To determine the TOF value at thermodynamic potential ( $0 \mathrm{~V}$ vs RHE), with the $j=j_{0}$, where $j_{0}$ is the exchange current densities of W/PdGa and $\mathrm{Pt} / \mathrm{PdGa}$, respectively.

\section{DATA AVAILABILITY}

The data generated during this study are available from the corresponding author on reasonable request. 


\section{CODE AVAILABILITY}

All code used to calculate the presented results is available from the corresponding author upon reasonable request.

Received: 16 June 2021; Accepted: 30 November 2021; Published online: 20 December 2021

\section{REFERENCES}

1. Rajamathi, C. R. et al. Weyl semimetals as hydrogen evolution catalysts. Adv. Mater 29, 1606202 (2017).

2. Li, L., Zeng, J., Qin, W., Cui, P. \& Zhang, Z. Tuning the hydrogen activation reactivity on topological insulator heterostructures. Nano Energy 58, 40-46 (2019).

3. Li, J. et al. Topological quantum catalyst: Dirac nodal line states and a potential electrocatalyst of hydrogen evolution in the TiSi family. Sci. China Mater 61, 23-29 (2018).

4. Chen, H., Zhu, W., Xiao, D. \& Zhang, Z. CO oxidation facilitated by robust surface states on Au-covered topological insulators. Phys. Rev. Lett 107, 056804 (2011).

5. Xiao, J. P., Kou, L. Z., Yam, C. Y., Frauenheim, T. \& Yan, B. H. Toward rational design of catalysts supported on a topological insulator substrate. ACS Catal $\mathbf{5}$, 7063-7067 (2015).

6. Yang, Q. et al. Topological engineering of Pt-group-metal-based chiral crystals toward high-efficiency hydrogen evolution catalysts. Adv. Mater. 32, 1908518 (2020).

7. $\mathrm{Li}, \mathrm{G}$. et al. Surface states in bulk single crystal of topological semimetal $\mathrm{Co}_{3} \mathrm{Sn}_{2} \mathrm{~S}_{2}$ toward water oxidation. Sci. Adv. 5, eaaw9867 (2019).

8. Schröter, N. B. et al. Observation and control of maximal chern numbers in a chiral topological semimetal. Science 369, 179-183 (2020).

9. Yao, M. et al. Observation of giant spin-split fermi-arc with maximal chern number in the chiral topological semimetal PtGa. Nat. Commun. 11, 1-7 (2020).

10. Rao, Z. et al. Observation of unconventional chiral fermions with long fermi arcs in CoSi. Nature 567, 496 (2019).

11. Tauster, S., Fung, S. \& Garten, R. L. Strong metal-support interactions. group 8 noble metals supported on titanium dioxide. J. Am. Chem. Soc. 100, 170-175 (1978).

12. Han, B. et al. Strong metal-support interactions between Pt single atoms and $\mathrm{TiO}_{2}$. Angewandte Chemi 132, 11824-11829 (2020).

13. Liu, L. \& Corma, A. Metal catalysts for heterogeneous catalysis: from single atoms to nanoclusters and nanoparticles. Chem. Rev. 118, 4981-5079 (2018).

14. Chen, J. G. Tuning catalytic properties using an atomically dispersed meta overlayer on transition metal carbide substrates. Natl. Sci. Rev. 4, 788-789 (2017).

15. Hou, Y. et al. Unraveling the reactivity and selectivity of atomically isolated metalnitrogen sites anchored on porphyrinic triazine frameworks for electroreduction of $\mathrm{CO}_{2}$. CCS Chem 1, 384-395 (2019).

16. Zhang, M., Guan, J., Tu, Y., Wang, S. \& Deng, D. Highly efficient conversion of surplus electricity to hydrogen energy via polysulfides redox. The Innov. 2, 100144 (2021)

17. Ivanova, A. et al. Metal-support interactions in $\mathrm{Pt} / \mathrm{Al}_{2} \mathrm{O}_{3}$ and $\mathrm{Pd} / \mathrm{Al}_{2} \mathrm{O}_{3}$ catalysts for CO oxidation. Appl. Catal. B-Environ. 97, 57-71 (2010).

18. van Deelen, T. W., Mejía, C. H. \& de Jong, K. P. Control of metal-support interactions in heterogeneous catalysts to enhance activity and selectivity. Nat. Catal. 2, 1-16 (2019).

19. Yang, D., Zhu, Q. \& Han, B. Electroreduction of $\mathrm{CO}_{2}$ in ionic liquid-based electrolytes. The Innov. 1, 100016 (2020).

20. Cheng, Y., Yang, S., Jiang, S. P. \& Wang, S. Supported single atoms as new class of catalysts for electrochemical reduction of carbon dioxide. Small Methods $\mathbf{3}$, 1800440 (2019).

21. He, Q. L., Lai, Y. H., Lu, Y., Law, K. T. \& Sou, I. K. Surface reactivity enhancement on a $\mathrm{Pd} / \mathrm{Bi}_{2} \mathrm{Te}_{3}$ heterostructure through robust topological surface states. Sci. Rep $\mathbf{3}$, 1-8 (2013).

22. Qu, Q. et al. Expediting hydrogen evolution through topological surface states on $\mathrm{Bi}_{2} \mathrm{Te}_{3}$. ACS Catal. 10, 2656-2666 (2020).

23. Rajamathi, C. R. et al. Photochemical water splitting by bismuth chalcogenide topological insulators. ChemPhysChem 18, 2322-2327 (2017).

24. Sessi, P. et al. Handedness-dependent quasiparticle interference in the two enantiomers of the topological chiral semimetal PdGa. Nat. Commun. 11, 3507 (2020).

25. Greeley, J., Jaramillo, T. F., Bonde, J., Chorkendorff, I. \& Nørskov, J. K. Computational high-throughput screening of electrocatalytic materials for hydrogen evolution. Nat. Mater. 5, 909-913 (2006)

26. Chen, W. et al. Rational design of single molybdenum atoms anchored on $\mathrm{N}$-doped carbon for effective hydrogen evolution reaction. Angew. Chem. Int. Ed. 56, 16086-16090 (2017).
27. Zhang, J. et al. Single platinum atoms immobilized on an mxene as an efficient catalyst for the hydrogen evolution reaction. Nat. Catal. 1, 985-992 (2018).

28. Chen, C. et al. Ruthenium-based single-atom alloy with high electrocatalytic activity for hydrogen evolution. Adv. Energy Mater. 9, 1803913 (2019).

29. Mathew, K., Sundararaman, R., Letchworth-Weaver, K., Arias, T. \& Hennig, R. G. Implicit solvation model for densityfunctional study of nanocrystal surfaces and reaction pathways. J. Chem. Phys. 140, 084106 (2014).

30. Nørskov, J. K. et al. Trends in the exchange current for hydrogen evolution. J. Electrochem. Soc. 152, J23-J26 (2005).

31. Wang, Y. et al. Adsorption-energy-based activity descriptors for electrocatalysts in energy storage applications. Natl. Sci. Rev. 5, 327-341 (2018).

32. Wang, $P$. et al. Precise tuning in platinum-nickel/nickel sulfide interface nanowires for synergistic hydrogen evolution catalysis. Nat. Commun. 8, 1-9 (2017).

33. Seh, Z. W. et al. Combining theory and experiment in electrocatalysis: Insights into materials design. Science. 355, eaad4998 (2017).

34. Greiner, M. T. et al. Free-atom-like $d$ states in single-atom alloy catalysts. Nat. Chem. 10, 1008-1015 (2018).

35. Fang, S. et al. Uncovering near-free platinum single-atom dynamics during electrochemical hydrogen evolution reaction. Nat. Commun. 11, 1-8 (2020).

36. Sanville, E., Kenny, S. D., Smith, R. \& Henkelman, G. Improved grid-based algorithm for bader charge allocation. J. Comput. Chem. 28, 899-908 (2007).

37. Yang, Y. et al. O-coordinated W-Mo dual-atom catalyst for $\mathrm{pH}$-universal electrocatalytic hydrogen evolution. Sci. Adv. 6, eaba6586 (2020).

38. Qiao, B. et al. Single-atom catalysis of $\mathrm{CO}$ oxidation using $\mathrm{Pt}_{1} / \mathrm{FeOx}$. Nat. Chem. 3 634-641 (2011)

39. Zhang, Z. et al. The simplest construction of single-site catalysts by the synergism of micropore trapping and nitrogen anchoring. Nat. Commun. 10, 1-7 (2019).

40. Yuan, Y. et al. $\mathrm{Co}_{3} \mathrm{Mo}_{3} \mathrm{~N}-\mathrm{An}$ efficient multifunctional electrocatalyst. The Innov. $\mathbf{2}$, 100096 (2021).

41. Zhang, $\mathrm{H}$. et al. Dynamic traction of lattice-confined platinum atoms into mesoporous carbon matrix for hydrogen evolution reaction. Sci. Adv. 4, eaao6657 (2018).

42. Luo, Z. et al. Chemically activating $\mathrm{MoS}_{2}$ via spontaneous atomic palladium interfacial doping towards efficient hydrogen evolution. Nat. Commun. 9, 1-8 (2018).

43. Yin, X.-P. et al. Engineering the coordination environment of single-atom platinum anchored on graphdiyne for optimizing electrocatalytic hydrogen evolution. Angew. Chem. Int. Ed. 57, 9382-9386 (2018).

44. Chen, W. et al. Single tungsten atoms supported on MOF-derived N-doped carbon for robust electrochemical hydrogen evolution. Adv. Mater. 30, 1800396 (2018).

45. Fei, H. et al. Atomic cobalt on nitrogen-doped graphene for hydrogen generation Nat. Commun. 6, 1-8 (2015)

46. Chen, W. et al. Rational design of single molybdenum atoms anchored on $\mathrm{N}$-doped carbon for effective hydrogen evolution reaction. Angew. Chem. Int. Ed. 56, 16086-16090 (2017).

47. $\mathrm{Li}, \mathrm{G}$. et al. In situ modification of a delafossite-type $\mathrm{PdCoO}_{2}$ bulk single crystal for reversible hydrogen sorption and fast hydrogen evolution. ACS Energy Lett. 4, 2185-2191 (2019).

48. Su, Y.-Q. et al. Stability of heterogeneous single-atom catalysts: a scaling law mapping thermodynamics to kinetics. Npj Comput. Mater. 6, 1-7 (2020).

49. Kresse, G. \& Furthmüller, J. Efficiency of ab-initio total energy calculations for metals and semiconductors using a plane-wave basis set. Comput. Mater. Sci. 6 15-50 (1996).

50. Kresse, G. \& Furthmüller, J. Efficient iterative schemes for ab initio total-energy calculations using a plane-wave basis set. Phys. Rev. B 54, 11169 (1996).

51. Kresse, G. \& Joubert, D. From ultrasoft pseudopotentials to the projector augmented-wave method. Phys. Rev. B 59, 1758 (1999).

52. Perdew, J. P., Burke, K. \& Ernzerhof, M. Generalized gradient approximation made simple. Phys. Rev. Lett. 77, 3865 (1996)

53. Grimme, S., Antony, J., Ehrlich, S. \& Krieg, H. A consistent and accurate ab initio parametrization of density functional dispersion correction (DFT-D) for the 94 elements H-Pu. J. Chem. Phys. 132, 154104 (2010).

54. Henkelman, G., Uberuaga, B. P. \& Jónsson, H. A climbing image nudged elastic band method for finding saddle points and minimum energy paths. J. Chem. Phys. 113, 9901-9904 (2000).

55. Chen, Z., Zhao, J., Cabrera, C. R. \& Chen, Z. Computational screening of efficient single-atom catalysts based on graphitic carbon nitride $\left(g-C_{3} N_{4}\right)$ for nitrogen electroreduction. Small Methods 3, 1800368 (2019).

56. $\mathrm{Ma}, \mathrm{D}$. et al. $3 d$ transition metal embedded $\mathrm{C}_{2} \mathrm{~N}$ monolayers as promising singleatom catalysts: a first-principles study. Carbon 105, 463-473 (2016). 


\section{ACKNOWLEDGEMENTS}

This work was financially supported by the European Research Council (ERC Advanced Grant No. 742068 T'OPMAT)'. We also acknowledge funding by the DFG through SFB 1143 (project ID. 247310070) and the Würzburg-Dresden Cluster of Excellence on Complexity and Topology in Quantum Matter ct.qmat (EXC2147, project ID. 39085490) and via DFG project HE 3543/35-1. JC Rao acknowledges the support of the Maryland NanoCenter and its AIM Lab. Some of our calculations are carried on the cluster of MPCDF, Max Planck Society.

\section{AUTHOR CONTRIBUTIONS}

Q.Y. designed the study, performed all theoretical calculations and analysis. G.L. performed material synthesis, characterizations, electrochemical measurements, and analysis with the help of Y.Z. and J.L. J.R. carried out the TEM and EDS experiments. Q. Y. and G.L. conceived the idea and co-wrote the manuscript. Y.S. and T.H. modified the manuscript. The project was supervised by C.F. All authors discussed the results and commented on the manuscript.

\section{FUNDING}

Open Access funding enabled and organized by Projekt DEAL.

\section{COMPETING INTERESTS}

The authors declare no competing interests.

\section{ADDITIONAL INFORMATION}

Supplementary information The online version contains supplementary material available at https://doi.org/10.1038/s41524-021-00684-5.

Correspondence and requests for materials should be addressed to Guowei Li or Yan Sun.

Reprints and permission information is available at http://www.nature.com/ reprints

Publisher's note Springer Nature remains neutral with regard to jurisdictional claims in published maps and institutional affiliations. Attribution 4.0 International License, which permits use, sharing, adaptation, distribution and reproduction in any medium or format, as long as you give appropriate credit to the original author(s) and the source, provide a link to the Creative Commons license, and indicate if changes were made. The images or other third party material in this article are included in the article's Creative Commons license, unless indicated otherwise in a credit line to the material. If material is not included in the article's Creative Commons license and your intended use is not permitted by statutory regulation or exceeds the permitted use, you will need to obtain permission directly from the copyright holder. To view a copy of this license, visit http://creativecommons. org/licenses/by/4.0/.

(c) The Author(s) 2021 